\title{
A Hybrid Readout System for the ATLAS TileCal Phase 2 Upgrade Demonstrator
}

\author{
Christian Bohm, Senior Member, IEEE on behalf of the ATLAS Tile Calorimeter System
}

\begin{abstract}
The ATLAS Tile Calorimeter phase 2 upgrade demonstrator project aims at installing hybrid on-detector electronic systems replacing 1-4 adjacent TileCal electronics drawers in ATLAS starting at the end of the long shut down of LHC 2013 to 2014. The new drawers will combine a fully functional phase 2 system with circuitry making them compatible with the present system. In the design we have emphasized redundancy and reliability. Data from and commands to the calorimeter are transferred via high speed $(5$ or $10 \mathrm{~Gb} / \mathrm{s}$ ) optical links.
\end{abstract}

\section{INTRODUCTION}

A present data from the hadronic Tile calorimeter [1] of the A ATLAS experiment [2] are stored in pipeline buffers on the detector and only read out if the event is accepted by the first level trigger. The higher luminosities from the planned accelerator upgrades will generate accumulated events that require more competent algorithms in the first level trigger. These algorithms will require more detailed information. The aim of the phase 2 upgrade (2022-23) of the Tile calorimeter is to digitally read out all detector information to the offdetector electronics in the counting room, USA-15, with minimum latency, and to do this reliably by including sufficient redundancy and radiation tolerance. There is a detailed plan to develop the final scheme for the phase II upgrade $\mathrm{R} \& \mathrm{D}$ activities covering all electronic detector components and good progress is made following this plan. To evaluate the technical solutions, a hybrid system will be built, combining the upgraded drawer electronics with parts of the present readout so that it may seamlessly replace a present module in ATLAS. This experience will give valuable input for the final design of the upgraded on- and off-detector electronics.

\section{A HYBRID READOUT SYSTEM}

The Tile calorimeter is a cylindrical assembly of $4 \times 64$ wedges. The base of these wedges houses extractable drawers, "SuperDrawers", containing the on-detector electronics. Thus, there are totally 256 SuperDrawers - 128 in the central barrel and 128 in extended barrels.

Manuscript received November 16, 2012

C. Bohm is with Stockholm University, Stockholm, SE 10691 Sweden (telephone: +46 5537 8696, e-mail: bohm@physto.se).
Each SuperDrawer can serve 48 PMTs, but only 45 are needed for the barrel and 32 for the extended barrel. In total we have close to 10000 PMT channels.

\section{A. The Present Readout System}

Each PMT is read out by front-end, "3-in-1”, boards [3]. In the present system groups of 6 3-in-1 outputs are received, digitized and pipelined by one "Digitizer" board (Fig 1a). Control, configuration and timing signals, and particularly the $40 \mathrm{MHz}$ LHC machine clock and the "Level 1 accept (L1A)" command, are supplied via optical fibers from the Time, Trigger and Control (TTC) system. At each L1A, all digitizers are read out via the "Interface" board and fiber links to Optical Receiver Boards and Read Out Drivers (RODs) in the counting room.

The name 3-in-1 alludes to the fact that the front-end boards, apart from reading out data, need to provide well defined charge injection pulses for calibration. A third function is to read out the PMT current caused by a calibration Cs-source that can be moved hydraulically from PMT to PMT for calibration. This is the "integrator" function.

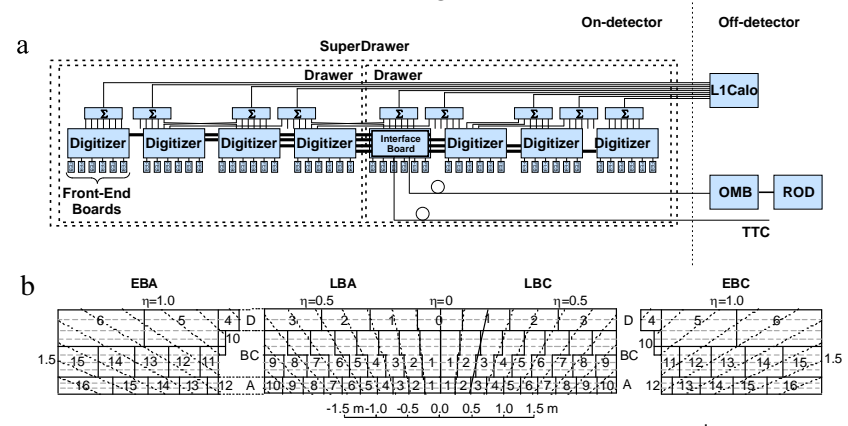

Fig. 1a) Block diagram of the present drawer electronics. b) A cross section of the TileCal detector showing the cell and trigger tower arrangement.

Light output from each calorimeter cell is read out independently by two PMTs, one on each side. The analog outputs from the calorimeter cells are also summed via special adder boards, in up to 9 trigger towers per drawer (Fig. 1a and b). The analog tower sums are then sent via electrical cables to the counting room and the first level trigger.

\section{B. The Upgraded Readout System}

The upgraded system (Fig. 2a) requires high bandwidth bidirectional links to transfer all data off the detector. The down link is used to provide TTC information, i.e. clocks, 
configurations and commands. L1A is no longer needed since all data are read out, and neither are the analog trigger outputs. Trigger information will be sent digitally to the first level trigger from the sRODs in USA-15.

Since redundancy and fault containment are of utmost importance to achieve maximum reliability, the new drawer electronics will be split into four independent units operating in parallel.

The main components of an upgraded drawer are:

- Front-End boards - three alternative designs

o Modified 3-in-1 (modification of existing FE)

o FE-ASIC (a front-end ASIC including ADCs)

o QIE (a multirange charge converter)

- MainBoards - digitizing the FE signals, if needed

- Link Daughter Board - control, data formatting and high speed communication

- High Voltage Power Supplies (HVPS) and voltage dividers

- Low Voltage Power Supplies (LVPS)

- Drawer mechanics - Standard or mini drawers

- Off-detector - sROD modules.

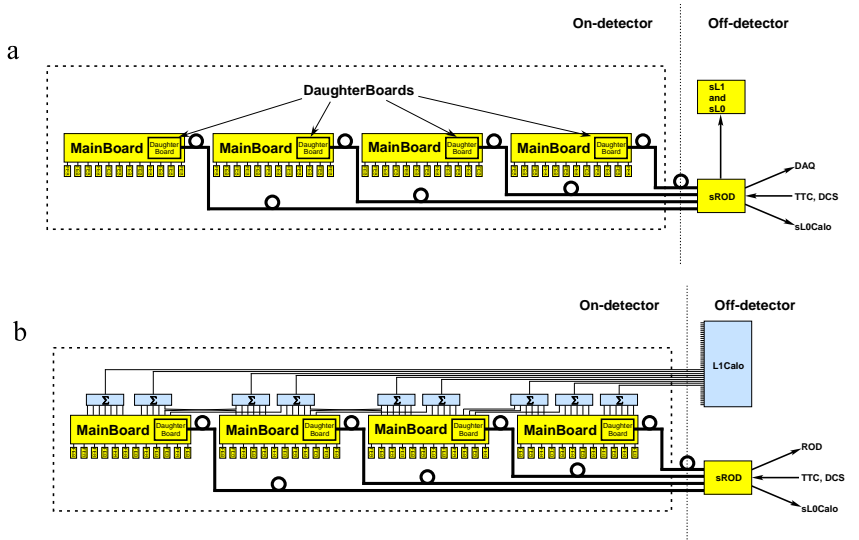

Fig. 2. Block diagram of the upgraded a) and hybrid b) drawer electronics.

\section{The Hybrid Readout System}

The new drawer design (Fig. 2b) can be made compatible with present system by retaining the analog summation circuitry and the trigger cables.

In this case, the sRODs will have to interpret and transfer the TTC information. They will also provide output data in a shape suitable for the present ROD processing as well as providing phase 2 trigger data for tests and evaluation (sL1Calo).

\section{THE UPGRADED SYSTEM COMPONENTS}

All on- and off-detector electronics must be replaced in the new system and most of it needs to be completely re-designed.

\section{A. The Drawer mechanics}

The present on-detector electronics are installed in "Superdrawers", each composed of two half-length "drawers" (see above).

The modularity of the upgraded on-detector electronics will allow half sized drawers, or "mini-drawers" (Fig. 3a). The advantage would be easier access and reduced demand for wide detector opening when servicing the Tile electronics. Due to the limited space available for opening the detector, this means that TileCal service work can be combined with other activities requiring opening. An ambitious program to study pros and cons has been initiated.

It is important to study the mini-drawer insertion and extraction. Tools for insertion and extraction have been developed and methods to prevent “zigzagging” during insertion as well as alignment precision have been studied. Other issues being studied concern module services: e.g. the design of reliable cooling water connections.

\section{B. Front-end Electronics}

Three different front-end alternatives are being considered for the upgraded system.

A modified version of the current 3 -in-1 has been developed, still based on discrete components. It has proved sufficiently radiation hard, and will give improved precision compared to the present system. It will provide two signal ranges, high and low gain, with gain ratio of 64 .

The FE-ASIC alternative will implement most of the functionality, including the ADCs, in an ASIC. This design will use three gain ranges.

The QIE, finally, is a charge integrating ASIC, earlier versions of which have been successfully used at Fermilab experiments. It has also been selected for the CMS ECAL. Here, one of four ranges is selected and digitized by a 6-bit ADC.

All front-end alternatives will also provide charge injection and integrator functions.

Since the modified 3-in-1 most easily accommodates analog trigger readout it is the one chosen for the demonstrator project. However, the final front-end choice will be based on results from future test beam studies.

\section{Readout Electronics}

Since the demonstrator will use the modified 3-in-1 the corresponding MainBoard [4] will contain 12-bit ADCs for the digitization (Fig.3b). It will be designed as two independent symmetric parts - each part reading out all cells, one using the right side readout, the other the left side.

The MainBoard is connected via a 400 pin FMC-type connector to the DaughterBoard, which is responsible for control, data formatting and high speed communication. The main functionality will be tested with a new DaughterBoard prototype that has been manufactured.

The DaughterBoard (Fig. 4) maintains the redundant separation into two independent parts. The processing logic is 
contained in two Kintex 7 FPGAs. Here it is important to verify that sufficient radiation tolerance can be maintained. The Clock, Trigger and Control are obtained using the GBT $[5,6]$ protocol on the down link.

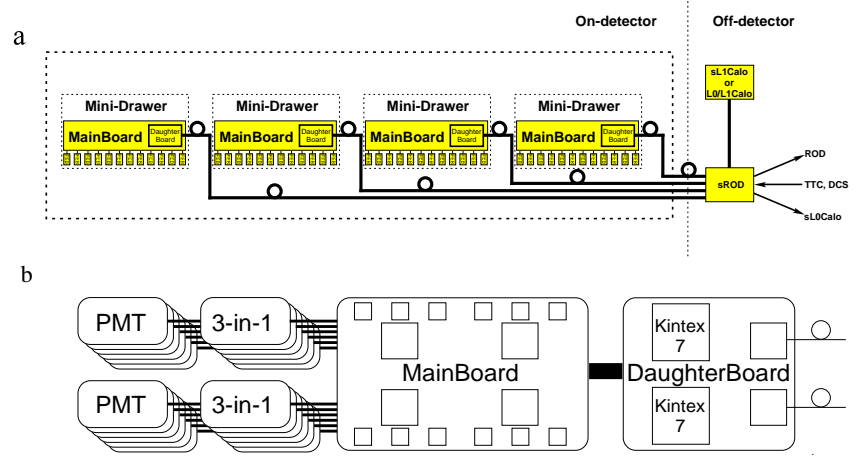

Fig. 3a) Block diagram of the mini-drawer arrangement. b) Block diagram of a drawer module.

For the links two different solutions are explored. One is based on a Avago laser and photo diode array transferring 5 Gb/s data over 12-fiber bundles. The Bit Error Rate (BER) is here about $10^{-12}$. The other solution employs a Luxtera 10 Gb/s QSFP modulator, which has high radiation tolerance and has proven highly reliable with a BER of $10^{-18}$ (according to the manufacturer). The choice will be made after thorough system tests.

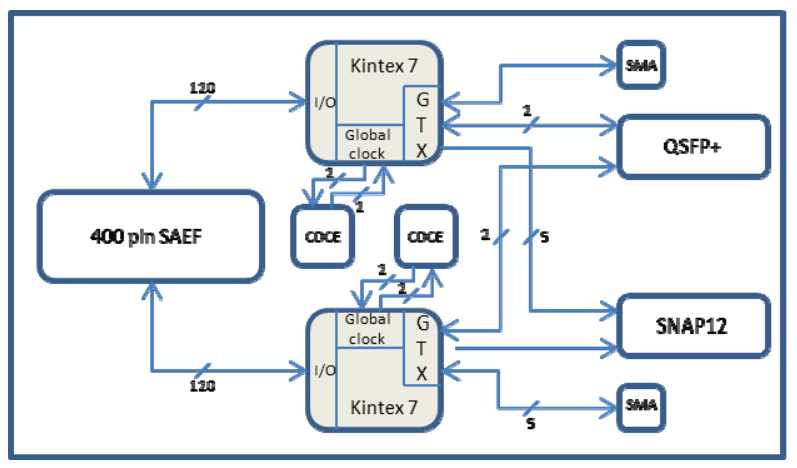

Fig. 4. Block diagram of the second prototype DaughterBoard.

\section{HV and PMT dividers}

For the HV regulation there are 6 different alternatives with different combination of reuse and new design. A decision on which alternative to choose will be made in the near future.

The higher luminosity will require larger divider currents to maintain PMT stability. For this purpose active dividers have been developed with active components in the last stages. These have proven to be linear even at higher luminosities. The old dividers had a non-linearity of about $1 \%$ at $3 \mu \mathrm{A}$ and about $2 \%$ at $7.5 \mu \mathrm{A}$ while the new divider has about $1 \%$ nonlinearity at $75 \mu \mathrm{A}$ and only $1.6 \%$ at about $150 \mu \mathrm{A}$. However, the radiation sensitivity of active parts still needs to be verified.

\section{E. LVPS}

The new low voltage power supplies will use a three stage power scheme with $200 \mathrm{~V}$ from the control room (USA-15) to local LVPS in the drawers. Boards receive down-regulated power at $10 \mathrm{~V}$, and Point Of Load (POL) regulators locally produce the required voltages. Diode coupling two independent $10 \mathrm{~V}$ supplies will provide increased redundancy. The aim is to use CERN developed rad-hard POL regulators when possible. However, $1 \mathrm{~V}$ and currents over $3 \mathrm{~A}$ are still problematic at the time of writing.

\section{F. Off-detector Electronics}

As mentioned above, the upgraded off-detector readout electronics [7] (sRODs) will need to take care of processing the trigger data. This includes both pulse timing identification and energy estimation. This is now done in the first level trigger pre-processor. The sRODs will also include the level 1 pipeline memories (which were previously performed in the detector), the present ROD functionality, and detector control. The sRODs will receive the detector information optically from the drawers, via either QSFP or ribbon fibers.

The Optical Link Card is a demonstrator for studying and gaining experience with multi-fiber high speed links. The card is now fully tested and allows transmission and reception of 12 optical fibers with GBT protocol at 4.8 Gbps (also tested with raw protocol at 6.25 Gbps)

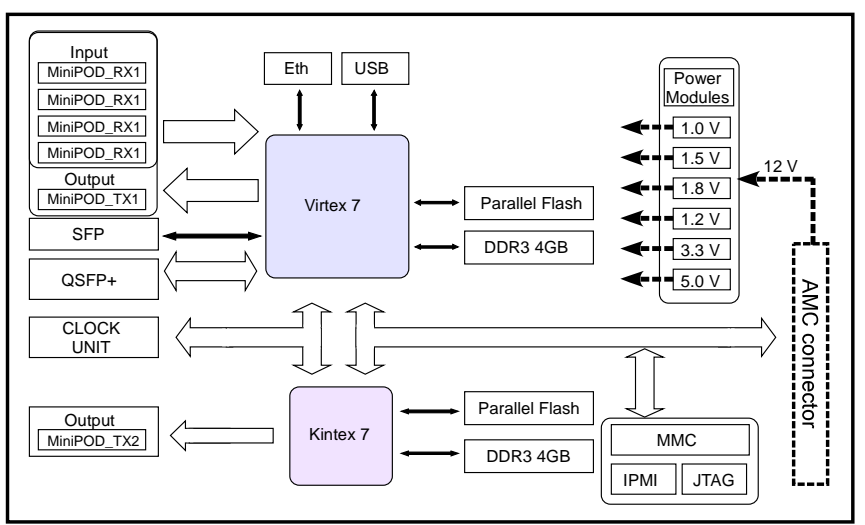

Fig. 5. Block diagram of the first sROD prototype.

A first sROD prototype (Fig. 5) will be manufactured early 2013. It will receive the data via MiniPOD connectors or via QSFP using the GBT protocol. The prototype will be based on the Xilinx FPGAs Virtex 7 and Kintex 7 and it will test pipeline, derandomizers, pulse identification, trigger algorithms and ROD processing as well as Detector Control and Monitoring.

\section{SCHEDULE}

The first installation of a demonstrator drawer is planned for the end of phase 0 shutdown i.e. mid 2014. If results are satisfactory, three more drawers will be installed in one of the 
following Christmas shutdowns. The phase 1 shutdown in 2018 is expected to provide a good opportunity to perform demonstrator modifications.

\section{Demonstrator Test Slice Development}

We have used Xilinx and ADC evaluation boards for early emulation of a readout slice in which the off-detector clock drives the on-detector electronics. When the on-detector evaluation board was replaced with the first MainBoard and DaughterBoard prototypes, the emulation could be made more detailed. The next steps involve using a second DaughterBoard and a second MainBoard prototype and in the end production versions of the on- and off-detector electronics will be used.

This succession of increasingly realistic emulation models allows successive development of the upgrade firmware and software.

\section{TEST System}

Most of the demonstrator functionality can be tested with existing test systems, Mobidick. The latest version Mobidick 4 [8] will be augmented with functionality for testing the digital path (sL1Calo).

\section{SUMMARY}

There is a development plan for the phase 2 upgrade of ATLAS Tile, where on-going R\&D activities aim at developing hardware, firmware and software. Some open questions remain to be answered, such as the choice of frontend boards, whether to use mini-drawers or not etc.. These will be resolved in due time.

The aim of the TileCal Demonstrator is to replace an existing drawer inside TileCal after proven to function according to specifications. There it will serve as an in-situ test bench for the new functionalities while delivering data into the present system.

Results have been positive so far with no show-stoppers foreseen, although much remains to be done before deployment in mid 2014.

\section{ACKNOWLEDGMENT}

This report summarizes a collective effort inside the Tile collaboration, more specifically those involved in the upgrade activities. The following persons have been directly involved: Gary Drake, Waruna Fernando, Bob Stanek, Mark Oreglia, Kelby Andersson, Fukun Tang, Alberto Valero, Fernando Carrio, Vicente Gonzáles, P. Moreno, Juan Valls, Daniel Eriksson, Steffen Muschter, Francois Vazeilles, R. Chadelas. R.Bonnefoy, G.Savinel, E.Sahuc, Carlos Solans and Irakli Minashvili.

\section{REFERENCES}

[1] ATLAS Collaboration, Readiness of the ATLAS Tile Calorimeter for LHC collisions, Eur. Phys. J. C 70 (2010) 1193
[2] ATLAS Collaboration, The ATLAS Experiment at the CERN Large Hadron Collider, JINST 3 (2008) S08003.

[3] K. Andersson et al. Design of the Front-end Analog Electronics for the ATLAS Tile Calorimeter, Nucl. Instrum. Meth. A 551 (2005) 469.

[4] S. Muschter, K. Andersson, C. Bohm, D. Eriksson, M. Oreglia,F. Tang, "Development of a readout link board for the TileCal phase 2 demonstrator", Proceedings of the Topical Workshop for Particle Physics, 2012.

[5] S. Baron, J. P. Cachemiche, F. Marin, P. Moreira, C. Soos, "Implementing the GBT data transmission protocol in FPGAs", TWEPP-09: Topical Workshop on Electronics for Particle Physics, 21 25 Sep 2009, pp. 631-635, Paris, France.

[6] P. Moreira, A. Marchioro, K. Kloukinas, “The GBT, a Proposed Architecture for Multi-Gb/s Data Transmission in High Energy Physics”, TWEPP-07: Topical Workshop on Electronics for Particle Physics, 3-7 September 2007, pp. 332-336, Prague, Czech Republic.

[7] F. Carrio, V. Castillo, A. Ferrer, L. Fiorini, V. Gonzalez, Y. Hernandez, E. Higon, P. Moreno, E. Sanchis, C. Solans, A. Valero, J. Valls, J., "Functional super Read-Out Driver demonstrator for the Phase 2 Upgrade of the ATLAS Tile Calorimeter", IEEE Nuclear Science Symposium and Medical Imaging Conference (NSS/MIC), vol., no., pp.167-170, 23-29 Oct. 2011.

[8] J. Alves, D. Calvet, F. Carrió, M. Crouau, K. Hee Yeun, I. Minashvili, S. Nemecek, G. Qin, V. Schetinno, C. Solans, G. Usai, A. Valero, "A new portable test bench for the ATLAS Tile Calorimeter front-end electronics", Proceedings of the Topical Workshop for Particle Physics, 2012. 